\title{
About improving integer multiplication in processors
}

The product of integers is one of the commonly used operations performed by modern computers. For example, when solving systems of equations, when working with vector graphics and other computing processes, the volume of multiplication operations can reach up to $30 . .50 \%$ of the total number of operations performed in ALU. If we consider the operation of adding integers, which is performed for one internal tick of the processor, then the operation of multiplication, when performed in the classical way and using sequential bit shifts and additions, will be performed for $\mathrm{N}$ or for $\mathrm{N} / 2$ (in the case of processing 2 bits at once) of internal ticks the processor. In the literature, it is noted that in modern processors the execution of this operation is optimized, however, specific details of how the optimized operation of multiplication is not published.

The classical algorithm for multiplying two numbers is shown in Fig. 1, where a bit shift to the left is performed sequentially at each step and, depending on the value, summation occurs. It works along next example, and if first integer is

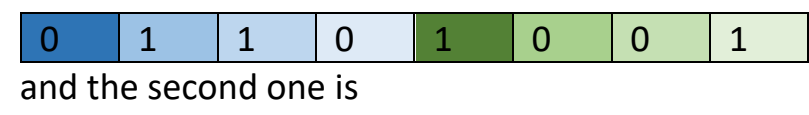

\begin{tabular}{|l|l|l|l|l|l|l|l|}
\hline 1 & 1 & 0 & 0 & 0 & 1 & 1 & 1 \\
\hline
\end{tabular}

The result will be gotten as the successive sum 8 same integers but with bit-shifts:

\begin{tabular}{|l|l|l|l|l|l|l|l|l|l|l|l|l|l|l|l|l|l|}
\hline & & & & & & & & & & 1 & 1 & 0 & 0 & 0 & 1 & 1 & 1 \\
\hline Step1 & 1 & & & & & & & & & 1 & 1 & 0 & 0 & 0 & 1 & 1 & 1 \\
\hline +step2 & 0 & & & & & & & & 0 & 0 & 0 & 0 & 0 & 0 & 0 & 0 & \\
\hline +step3 & 0 & & & & & & & 0 & 0 & 0 & 0 & 0 & 0 & 0 & 0 & & \\
\hline +step4 & 1 & & & & & & 1 & 1 & 0 & 0 & 0 & 1 & 1 & 1 & & & \\
\hline +step5 & 0 & & & & & 0 & 0 & 0 & 0 & 0 & 0 & 0 & 0 & & & & \\
\hline +step6 & 1 & & & & 1 & 1 & 0 & 0 & 0 & 1 & 1 & 1 & & & & & \\
\hline +step7 & 1 & & & 1 & 1 & 0 & 0 & 0 & 1 & 1 & 1 & & & & & & \\
\hline +step8 & 0 & & 0 & 0 & 0 & 0 & 0 & 0 & 0 & 0 & & & & & & & \\
\hline & Result & & 1 & 0 & 1 & 0 & 0 & 0 & 1 & 1 & 0 & 0 & 1 & 1 & 1 & 1 & 1 \\
\hline
\end{tabular}

Fig 1. Classical shifts scheme of multiplication

As mentioned above, this method is quite resource-intensive and runs in a loop, the number of iterations, which is proportional to the number of characters of one of the factors. The Karatsuba algorithm is widely known https://en.wikipedia.org/wiki/Karatsuba_algorithm by which the complexity of the main process is reduced by factoring into components and processing, which in case of parallelization can have a significant effect and reduce internal processor tics. In general, this method is similar to the product of polynomials, in the simplest case, the product of two binomials. 
In the pursuit of productivity and reducing the resource intensity of operations, the question arises: is it possible to reduce the number of internal operations performed in ALU? As an option to increase productivity and reduce the execution time of an integer multiplication, we propose a partition of factors into elementary components and the use of an auxiliary multiplication table, so if $A=A 0+A 1^{*} 2^{\wedge} 4$ and $B=B 0+B 1^{*} 2^{\wedge} 4$ then $A * B=A 0 * B 0+\left(A 0 * B 1+A 1 * B 0 * 2^{\wedge} 4\right.$ $+\mathrm{A} 1 * \mathrm{~B}^{*} 2^{\wedge} 8$.

Most computer processors today operate with 32 or 64-bits integers, so to obtain a positive effect of multiplication integers should be parted on parts that easily form back 32 or 64 . But there is should be considered size of the auxiliary multiplication table. If use 8-bits elements and the auxiliary multiplication table size 256 on 256 then instead of multiplication that could be cost upto 32 additions of factors.

Consider this method by the example of multiplying two 8-bit numbers, which will be divided into 4-bit components and using an auxiliary table of size $16 * 16$. There are two factors $A$ and $B$, which are split into $A 0+A 1^{*} 2^{\wedge} 4$ and $B 0+B 1^{*} 2^{\wedge} 4$ where $A 1$ and $B 1$ are the highest 4 digits, and $A 0$ and $B 0$ are the lower 4 digits. The auxiliary table is shown in Fig. 4 , and as a result, instead of performing the multiplication operation, the operation of extracting the result from this table is performed. In fact, this auxiliary table is a linear array of Fig. 5, in which the product of two 4-digit numbers is stored in a cell with the address formed from the union of two 4-digit numbers. To achieve a positive effect, this table is located directly in the ALU itself, it is also easy to notice that this table is symmetrical relative to its main diagonal, and has the first row and column filled with zero values, and the second values equal to the address of the table cell, therefore the size of this table may be reduced by at least two.

In this example 8-bit integers are splitted out on 4-bit, but in real processors 32-bits or 64-bits integers should be splitted out on 8-bit or even 16-bit.

\begin{tabular}{|l|l|l|l|l|l|l|l|}
\hline \multicolumn{4}{|c|}{ A1 } & \multicolumn{5}{c|}{ A0 } \\
\hline 0 & 1 & 1 & 0 & 1 & 0 & 0 & 1 \\
\hline
\end{tabular}

Fig 2. Splitting of the first factor into subfactors

And the second one is

\begin{tabular}{|l|l|l|l|l|l|l|l|}
\hline \multicolumn{4}{|c|}{ B1 } & \multicolumn{5}{|c|}{ B0 } \\
\hline 1 & 1 & 0 & 0 & 0 & 1 & 1 & 1 \\
\hline
\end{tabular}

Fig 3. Splitting of the second factor into subfactors

\begin{tabular}{|c|c|c|c|c|c|c|c|c|c|c|c|c|c|c|c|c|c|}
\hline & & 0 & 1 & 2 & 3 & 4 & 5 & 6 & 7 & 8 & 9 & 10 & 11 & 12 & 13 & 14 & 15 \\
\hline & & 0000 & 0001 & 0010 & 0011 & 0100 & 0101 & 0110 & 0111 & 1000 & 1001 & 1010 & 1011 & 1100 & 1101 & 1110 & 1111 \\
\hline 0 & 0000 & 00000000 & 00000000 & 00000000 & 00000000 & 00000000 & 00000000 & 00000000 & 00000000 & 00000000 & 00000000 & 00000000 & 00000000 & $0 \mid 00000000$ & 00000000 & 000000000 & 00000000 \\
\hline 1 & 0001 & 00000000 & 00000001 & 00000010 & 00000011 & 00000100 & 00000101 & 00000110 & 00000111 & 00001000 & 00001001 & 00001010 & 00001011 & 100001100 & 00001101 & 100001110 & 00001111 \\
\hline
\end{tabular}




\begin{tabular}{|c|c|c|c|c|c|c|c|c|c|c|c|c|c|c|c|c|c|}
\hline 2 & 0010 & 000000 & 00000010 & 00000100 & 00000110 & 00001000 & 00001010 & 00001100 & 00001110 & 00010000 & 00010010 & 00010100 & 00010110 & 00011000 & 00011010 & 00011100 & 00011110 \\
\hline 3 & 0011 & 000000 & 00000011 & 00000110 & 00001001 & 00001100 & 00001111 & 00010010 & 00010101 & 00011000 & 00011011 & 00011110 & 00100001 & 00100100 & 00100111 & 100101010 & 00101101 \\
\hline 4 & 0100 & 0000000 & 00000100 & 00001000 & 00001100 & 00010000 & 00010100 & 00011000 & 00011100 & 00100000 & 00100100 & 00101000 & 00101100 & 00110000 & 00110100 & 00111000 & 00111100 \\
\hline 5 & 0101 & 0000000 & 00000101 & 00001010 & 00001111 & 00010100 & 00011001 & 00011110 & 00100011 & 00101000 & |00101101 & 00110010 & 00110111 & 00111100 & 01000001 & 101000110 & 01001011 \\
\hline 6 & 0110 & 00000000 & 00000110 & 00001100 & 00010010 & 00011000 & 00011110 & 00100100 & 00101010 & 00110000 & 00110110 & 00111100 & 01000010 & 01001000 & 01001110 & 01010100 & 01011010 \\
\hline 7 & 0111 & 0000000 & 00000111 & 00001110 & 00010101 & 00011100 & 00100011 & 00101010 & |00110001 & 00111000 & 00111111 & 01000110 & 01001101 & 01010100 & 01011011 & 101100010 & 01101001 \\
\hline 8 & 1000 & 00000000 & 00001000 & 00010000 & 00011000 & 00100000 & 00101000 & 00110000 & 00111000 & 01000000 & 01001000 & 01010000 & 01011000 & 01100000 & 01101000 & $0 \mid 01110000$ & 01111000 \\
\hline 9 & 1001 & 00000000 & 00001001 & 00010010 & 00011011 & 00100100 & 00101101 & 00110110 & |00111111 & 01001000 & 01010001 & 01011010 & 01100011 & 01101100 & 01110101 & 101111110 & 10000111 \\
\hline 10 & 1010 & ח000000 & 00001010 & 00010100 & 00011110 & 00101000 & 00110010 & 00111100 & 01000110 & 01010000 & 01011010 & 01100100 & 01101110 & 01111000 & 10000010 & $0 \mid 10001100$ & 10010110 \\
\hline 11 & 1011 & 00000000 & 00001011 & 00010110 & 00100001 & $00101100 \mid$ & 00110111 & 01000010 & |01001101 & 01011000 & 01100011 & | $01101110 \mid$ & 0111100101 & 10000100 & 10001111 & $1|10011010|$ & 10100101 \\
\hline 12 & 1100 & 00000000 & 00001100 & 00011000 & 00100100 & 00110000 & 00111100 & 01001000 & 01010100 & 01100000 & 01101100 & 01111000 & 10000100 & 10010000 & 10011100 & | 10101000 & 10110100 \\
\hline 13 & 1101 & 00000000 & 00001101 & 00011010 & 00100111 & 00110100 & 01000001 & 01001110 & 01011011 & 01101000 & 01110101 & 10000010 & 10001111 & $|10011100|$ & 10101001 & $1|10110110|$ & 11000011 \\
\hline 14 & 1110 & 00000000 & 00001110 & 00011100 & 00101010 & 00111000 & 01000110 & 01010100 & 01100010 & 01110000 & 01111110 & 10001100 & | 10011010 & $|10101000|$ & 10110110 & $0 \mid 11000100$ & 11010010 \\
\hline 15 & 1111 & 00000000 & 00001111 & 00011110 & 00101101 & 00111100 & 01001011 & 01011010 & 01101001 & 01111000 & 10000111 & 10010110 & 10100101 & $\mid 10110100$ & 11000011 & $1|11010010|$ & 11100001 \\
\hline
\end{tabular}

Fig.4. Example of auxiliary multiplication table $16 * 16$

To achieve an effective result the auxiliary table must be placed in the ALU at the same level as the processor's registers and results will be gotten at once from there instead of performing additions and shiftings. In fact, the auxiliary multiplication table is a symmetrical triangle table and the pink cells (on Fig. 4) have their twins, so its size can be easily reduced in twice.

In fact, this auxiliary multiplication table is a simple linear array in which the address is combined from parts of multipliers.

\begin{tabular}{|c|c|c|c|}
\hline A1 & B1 & Address & Result \\
\hline 0000 & 0001 & 00000001 & 00000000 \\
\hline \multicolumn{4}{|c|}{$\ldots \ldots}$. \\
\hline 0011 & 0000 & 00110000 & 00000000 \\
\hline 0011 & 0001 & 00110001 & 00000011 \\
\hline
\end{tabular}




\begin{tabular}{|l|l|l|l|}
\hline 0011 & 0010 & 00110010 & 00000110 \\
\hline 0011 & 0011 & 00110011 & 00001001 \\
\hline 0011 & 0100 & 00110100 & 00001100 \\
\hline 0011 & 0101 & 00110101 & 00001111 \\
\hline 0011 & 0110 & 00110110 & 00010010 \\
\hline 0011 & 0111 & 00110111 & 00010101 \\
\hline 0011 & 1000 & 00111000 & 00011000 \\
\hline 0011 & 1001 & 00111001 & 00011011 \\
\hline 0011 & 1010 & 00111010 & 00011110 \\
\hline 0011 & 1011 & 00111011 & 00100001 \\
\hline 0011 & 1100 & 00111100 & 00100100 \\
\hline 0011 & 1101 & 00111101 & 00100111 \\
\hline 0011 & 1110 & 00111110 & 00101010 \\
\hline 0011 & 1111 & 00111111 & 00101101 \\
\hline 0100 & 0000 & 01000000 & 00000000 \\
\hline 0100 & 0001 & 01000001 & 00000011 \\
\hline 0100 & 0010 & 01000010 & 00000110 \\
\hline 0100 & 0011 & 01000011 & 00001001 \\
\hline 0100 & 0100 & 01000100 & 00001100 \\
\hline & & $\ldots \ldots$ & \\
\hline 1111 & 1101 & 11111101 & 11000011 \\
\hline 1111 & 1110 & 11111110 & 11010010 \\
\hline 1111 & 1111 & 11111111 & 11100001 \\
\hline
\end{tabular}

Fig.5 


\begin{tabular}{|c|c|c|c|c|c|c|c|c|c|c|c|c|c|c|c|c|c|}
\hline & & 0 & 1 & 2 & 3 & 4 & 5 & 6 & 7 & 8 & 9 & 10 & 11 & 12 & 13 & 14 & 15 \\
\hline & & 0000 & 0001 & 0010 & 0011 & 0100 & 0101 & $1=0110$ & 0111 & 1000 & $A 2=1001$ & 1010 & 1011 & 1100 & 1101 & 1110 & 1111 \\
\hline 0 & 0000 & 00000000 & 00000000 & 00000000 & 00000000 & 00000000 & 00000000 & 00000000 & 00000000 & 00000000 & 00000000 & 00000000 & 00000000 & 00000000 & 00000000 & 00000000 & 00000000 \\
\hline 1 & 0001 & 00000000 & 00000001 & 00000010 & 00000011 & 00000100 & 00000101 & 00000110 & 00000111 & 00001000 & 00001001 & 00001010 & 00001011 & 00001100 & 00001101 & 00001110 & 00001111 \\
\hline 2 & 0010 & 00000000 & 00000010 & 00000100 & 00000110 & 00001000 & 00001010 & 00001100 & 00001110 & 00010000 & 00010010 & 00010100 & 00010110 & 00011000 & 00011010 & 00011100 & 00011110 \\
\hline 3 & 0011 & 00000000 & 00000011 & 00000110 & 00001001 & 00001100 & 00001111 & 00010010 & 00010101 & 00011000 & 00011011 & 00011110 & 00100001 & 00100100 & 00100111 & 00101010 & 00101101 \\
\hline 4 & 0100 & 00000000 & 00000100 & 00001000 & 00001100 & 00010000 & 00010100 & 00011000 & 00011100 & 00100000 & 00100100 & 00101000 & 00101100 & 00110000 & 00110100 & 00111000 & 00111100 \\
\hline 5 & 0101 & 00000000 & 00000101 & 00001010 & 00001111 & 00010100 & 00011001 & 00011110 & 00100011 & 00101000 & 00101101 & 00110010 & 00110111 & 00111100 & 01000001 & 01000110 & 01001011 \\
\hline 6 & 0110 & 00000000 & 00000110 & 00001100 & 00010010 & 00011000 & 00011110 & 00100100 & 00101010 & 00110000 & 00110110 & 00111100 & 01000010 & 01001000 & 01001110 & 01010100 & 01011010 \\
\hline 7 & $B 2=0111$ & 00000000 & 00000111 & 00001110 & 00010101 & 00011100 & 00100011 & 00101010 & 00110001 & 00111000 & 00111111 & 01000110 & 01001101 & 01010100 & 01011011 & 01100010 & 01101001 \\
\hline 8 & 1000 & 00000000 & 00001000 & 00010000 & 00011000 & 00100000 & 00101000 & 00110000 & 00111000 & 01000000 & 01001000 & 01010000 & 01011000 & 01100000 & 01101000 & 01110000 & 01111000 \\
\hline 9 & 1001 & 00000000 & 00001001 & 00010010 & 00011011 & 00100100 & 00101101 & 00110110 & 00111111 & 01001000 & 01010001 & 01011010 & 01100011 & 01101100 & 01110101 & 01111110 & 10000111 \\
\hline 10 & 1010 & 00000000 & 00001010 & 00010100 & 00011110 & 00101000 & 00110010 & 00111100 & 01000110 & 01010000 & 01011010 & 01100100 & 01101110 & 01111000 & 10000010 & 10001100 & 10010110 \\
\hline 11 & 1011 & 00000000 & 00001011 & 00010110 & 00100001 & 00101100 & 00110111 & 01000010 & 01001101 & 01011000 & 01100011 & 01101110 & 01111001 & 10000100 & 10001111 & 10011010 & 10100101 \\
\hline 12 & $B 1=1100$ & 00000000 & 00001100 & 00011000 & 00100100 & 00110000 & 00111100 & 01001000 & 01010100 & 01100000 & 01101100 & 01111000 & 10000100 & 10010000 & 10011100 & 10101000 & 10110100 \\
\hline 13 & 1101 & 00000000 & 00001101 & 00011010 & 00100111 & 00110100 & 01000001 & 01001110 & 01011011 & 01101000 & 01110101 & 10000010 & 10001111 & 10011100 & 10101001 & 10110110 & 11000011 \\
\hline 14 & 1110 & 00000000 & 00001110 & 00011100 & 00101010 & 00111000 & 01000110 & 01010100 & 01100010 & 01110000 & 01111110 & 10001100 & 10011010 & 10101000 & 10110110 & 11000100 & 11010010 \\
\hline 15 & 1111 & 00000000 & 00001111 & 00011110 & 00101101 & 00111100 & 01001011 & 01011010 & 01101001 & 01111000 & 10000111 & 10010110 & 10100101 & 10110100 & 11000011 & 11010010 & 11100001 \\
\hline
\end{tabular}

Fig.6. Example of extracting values for multiplication.

The operation of multiplying two integers for this example will cost 4 accesses to the auxiliary table and calculation of the result, and since the auxiliary table is constant, then these 4 calls can be performed simultaneously, as can be seen from the formula, the quantities $A 0 *$ B0 and A1* B1 are deliberately separated by 8 digits so they can be immediately grouped and placed as an argument to summarize step 1 , to which the values from cells with addresses $A 0 * B 1$ and $A 1 * B 0$, as a result, for the product, 3 additions are required instead of 8 shown in Fig. 1.

\begin{tabular}{|c|c|c|c|c|c|c|c|c|c|c|c|c|c|c|c|c|c|c|}
\hline Step1 & $\begin{array}{c}\mathrm{A} 0 * \mathrm{~B} 0+ \\
\mathrm{A} 1 * \mathrm{~B} 1\end{array}$ & F.overflow & 0 & 1 & 0 & 0 & 1 & 0 & 0 & 0 & 0 & 0 & 1 & 1 & 1 & 1 & 1 & 1 \\
\hline
\end{tabular}




\begin{tabular}{|c|c|c|c|c|c|c|c|c|c|c|c|c|c|c|c|c|c|c|}
\hline +Step2 & $\mathrm{A} 0 * \mathrm{~B} 1$ & & & & & & 0 & 0 & 1 & 0 & 1 & 0 & 1 & 0 & & & & \\
\hline +Step3 & $\mathrm{A} 1 * \mathrm{~B} 0$ & & & & & & 0 & 1 & 1 & 0 & 1 & 1 & 0 & 0 & & & & \\
\hline & result & $x$ & 0 & 1 & 0 & 1 & 0 & 0 & 0 & 1 & 1 & 0 & 0 & 1 & 1 & 1 & 1 & 1 \\
\hline
\end{tabular}

Fig.7. Calculating multiplication

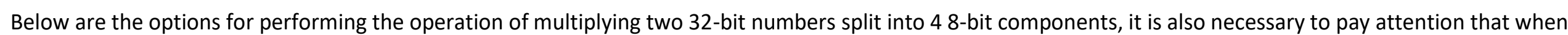
generating addresses for accessing the auxiliary table, very often only one half of the result address changes (highlighted in bold italics in Fig. 8), which also contributes to saving resources. As a result, instead of 32 consecutive additions, the multiplication in Fig. 8 requires 7 additions.

If 32-bit integers split on 8-bit items, then to multiple them $A=a 3^{*} 2^{\wedge} 24+a 2^{*} 2^{\wedge} 16+a 1^{*} 2^{\wedge} 8+a 0$ and $B=b 3^{*} 2^{\wedge} 24+b 2^{*} 2^{\wedge} 16+b 1^{*} 2^{\wedge} 8+a 0$. The result of their multiplication is

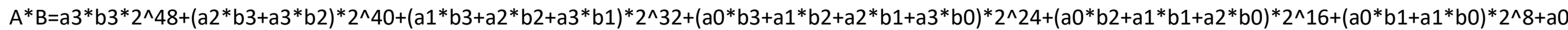
*b0.

\begin{tabular}{|c|c|c|c|c|c|c|c|c|c|}
\hline & F.overflow & $(64) 00000000$ & (56)00000000 & $(48) 00000000$ & $(40) 00000000$ & (32)00000000 & $(24) 00000000$ & (16)00000000 & (8)00000000 \\
\hline Factor a & & & & & & $\mathrm{A} 3$ & $\mathrm{~A} 2$ & A1 & $\mathrm{AO}$ \\
\hline Factor $b$ & & & & & & B3 & B2 & B1 & BO \\
\hline Step1 & & \multicolumn{2}{|c|}{ A3*B3 } & \multicolumn{2}{|c|}{ A3*B1 } & \multicolumn{2}{|c|}{$A 2 * B O$} & \multicolumn{2}{|c|}{$A 0 * B O$} \\
\hline +Step2 & & & \multicolumn{2}{|c|}{$A 2 * B 3$} & \multicolumn{2}{|c|}{$A 0 * B 3$} & \multicolumn{2}{|c|}{$A 0 * \mathrm{~B} 1$} & \\
\hline +Step3 & & & \multicolumn{2}{|c|}{$A 3 * B 2$} & \multicolumn{2}{|c|}{$A 1 * B 2$} & \multicolumn{2}{|c|}{$A 1 * B 0$} & \\
\hline +Step4 & & & & \multicolumn{2}{|c|}{$A 2 * B 2$} & \multicolumn{2}{|c|}{$\mathrm{A} 1 * \mathrm{~B} 1$} & & \\
\hline +Step5 & & & & \multicolumn{2}{|c|}{$\mathrm{A} 1 * \mathrm{~B} 3$} & \multicolumn{2}{|c|}{$A 0 * B 2$} & & \\
\hline +Step6 & & & & & \multicolumn{2}{|c|}{$\mathrm{A} 2 * \mathrm{~B} 1$} & & & \\
\hline +Step7 & & & & & \multicolumn{2}{|c|}{$\mathrm{A} 3 * \mathrm{~B} 0$} & & & \\
\hline RESULT & & & & & & & & & \\
\hline
\end{tabular}

Pic 8 Performing 32-bit integer multiplication without optimization

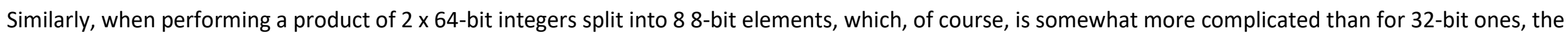
cost of the multiplication operation will be 15 instead of 64 additions. $A=a 7^{*} 2^{\wedge} 56+a 6^{*} 2^{\wedge} 48+a 5^{*} 2^{\wedge} 40+a 4^{*} 2^{\wedge} 32+a 3^{*} 2^{\wedge} 24+a 2^{*} 2^{\wedge} 16+a 1^{*} 2^{\wedge} 8+a 0$ and $B=$ $b 7^{*} 2^{\wedge} 56+b 6^{*} 2^{\wedge} 48+b 5^{*} 2^{\wedge} 40+b 4^{*} 2^{\wedge} 32+b 3^{*} 2^{\wedge} 24+b 2 * 2^{\wedge} 16+b 1^{*} 2^{\wedge} 8+b 0$

$A * B=a 7^{*} b 7^{*} 2^{\wedge} 112+\left(a 7^{*} b 6+a 6^{*} b 7\right) * 2^{\wedge} 104+\left(a 7^{*} b 5+a 6^{*} b 6+a 5^{*} b 7\right) * 2^{\wedge} 96+(a 7 * b 4+a 6 * b 5+$

$\left.a 5^{*} b 6+a 4^{*} b 7\right)^{*} 2^{\wedge} 88+\left(a 7^{*} b 3+a 6^{*} b 5+a 5^{*} b 5+a 4 * b 6+a 3 * b 7\right) * 2^{\wedge} 80+\left(a 7 * b 2+a 6 * b 3+a 5 * b 4+a 4 * b 5+a 3 * b 6+a 2^{*} b 7\right) * 2 \wedge 72+$ 
$+(a 7 * b 1+a 6 * b 2+a 5 * b 3+a 4 * b 4+a 3 * b 5+a 2 * b 6+a 1 * b 7) * 2 \wedge 64+$

$\left(a 7^{*} b 0+a 6 * b 1+a 5 * b 2+a 4 * b 3+a 3 * b 4+a 2 * b 5+a 1 * b 6+a 0 * b 7\right) * 2 \wedge 56+(a 6 * b 0+a 5 * b 1+a+b 2+a 3 * b 3+a 2 * b 4+a 1 * b 5+a 0 * b 6) * 2 \wedge 48+$

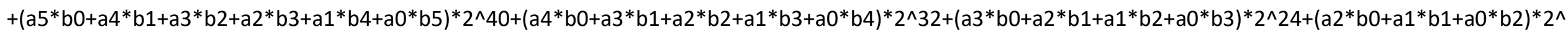
$16+(a 1 * b 0+a 0 * b 1) * 2 \wedge 8+a 0 * b 0$

\begin{tabular}{|c|c|c|c|c|c|c|c|c|c|c|}
\hline & $(128) 00000000$ & $(120) 00000000$ & $(112) 00000000$ & $(104) 00000000$ & (96)00000000 & $(88) 00000000$ & $(80) 00000000$ & $(72) 00000000$ & (64)00000000 & $(56) 00000$ \\
\hline Factor a & & & & & & & & & A7 & A6 \\
\hline Factor b & & & & & & & & & B7 & B6 \\
\hline Step1 & \multicolumn{2}{|c|}{$A 7 * B 7$} & \multicolumn{2}{|c|}{ A7*B5 } & \multicolumn{2}{|c|}{$\mathrm{A} 7 * \mathrm{~B} 3$} & \multicolumn{2}{|c|}{$\mathrm{A} 7 * \mathrm{~B} 1$} & \multicolumn{2}{|c|}{$\mathrm{A} 6 * \mathrm{~B} 0$} \\
\hline +Step2 & & \multicolumn{2}{|c|}{$\mathrm{A} 7 * \mathrm{~B} 6$} & \multicolumn{2}{|c|}{$\mathrm{A} 7{ }^{*} \mathrm{~B} 4$} & \multicolumn{2}{|c|}{$\mathrm{A} 7 * \mathrm{~B} 2$} & \multicolumn{2}{|c|}{$\mathrm{A} 7{ }^{*} \mathrm{~B} 0$} & \\
\hline +Step3 & & \multicolumn{2}{|c|}{$A 6 * B 7$} & \multicolumn{2}{|c|}{$\mathrm{A} 6 * \mathrm{~B} 5$} & \multicolumn{2}{|c|}{$\mathrm{A6*B3}$} & \multicolumn{2}{|c|}{$\mathrm{A} 6 * \mathrm{~B} 1$} & \\
\hline +Step4 & & & \multicolumn{2}{|c|}{$\mathrm{A} 6 * \mathrm{~B} 6$} & \multicolumn{2}{|c|}{$\mathrm{A} 6 * \mathrm{~B} 4$} & \multicolumn{2}{|c|}{$A 6 * B 2$} & \multicolumn{2}{|c|}{$A 4 * B 2$} \\
\hline +Step5 & & & \multicolumn{2}{|c|}{$A 5 * B 7$} & \multicolumn{2}{|c|}{$A 5 * B 5$} & \multicolumn{2}{|c|}{$A 5 * B 3$} & \multicolumn{2}{|c|}{$A 5 * B 1$} \\
\hline +Step6 & & & & \multicolumn{2}{|c|}{$\mathrm{A} 5 * \mathrm{~B} 6$} & \multicolumn{2}{|c|}{$\mathrm{A} 5^{*} \mathrm{~B} 4$} & \multicolumn{2}{|c|}{$\mathrm{A} 5 * \mathrm{~B} 2$} & \\
\hline +Step7 & & & & \multicolumn{2}{|c|}{$\mathrm{A} 4 * \mathrm{~B} 7$} & \multicolumn{2}{|c|}{$\mathrm{A} 4 * \mathrm{~B} 5$} & \multicolumn{2}{|c|}{$\mathrm{A} 4 * \mathrm{~B} 3$} & \\
\hline +Step8 & & & & & \multicolumn{2}{|c|}{$\mathrm{A} 4 * \mathrm{~B} 6$} & \multicolumn{2}{|c|}{$\mathrm{A} 4 * \mathrm{~B} 4$} & \multicolumn{2}{|c|}{$\mathrm{A} 2 * \mathrm{~B} 4$} \\
\hline +Step9 & & & & & \multicolumn{2}{|c|}{$\mathrm{A} 3 * \mathrm{~B} 7$} & \multicolumn{2}{|c|}{$\mathrm{A} 3 * \mathrm{~B} 5$} & \multicolumn{2}{|c|}{$A 3 * B 3$} \\
\hline +Step10 & & & & & & $A 3^{*}$ & B6 & A3 & & \\
\hline +Step11 & & & & & & $A 2^{*}$ & B7 & $\mathrm{A} 2$ & $\mathrm{~B} 5$ & \\
\hline +Step12 & & & & & & & $A 2$ & B6 & A0 & *B6 \\
\hline +Step13 & & & & & & & A1 & B7 & A1 & *B5 \\
\hline +Step14 & & & & & & & & $\mathrm{A} 1$ & B7 & \\
\hline +Step15 & & & & & & & & $\mathrm{A} 1$ & B6 & \\
\hline RESULT & & & & & & & & & & \\
\hline
\end{tabular}

Pic 9. Performing 64-bit integer multiplication without optimization

It is easy to see that far from the entire width of the adder is used at 100\%, and Figure 9 shows that starting from step 6, a large amount of unused space appears, at stages 4 and 5 up to 32 bits or at least 24, at 6 and 7 at least 40 digits, and at least 56 at 8 and 9, which may allow you to combine the implementation of several steps in one. So, for example, steps 14 and 15 can be performed together with 4 and 5, 12 and 13 together with 6 and 7 , etc. Figure 10 shows an embodiment of the multiplication, more efficiently using the entire width of the adder and allowing to reduce the number of addition operations to 10 or even to 9 . Thus, the method of performing a product using the auxiliary multiplication table allows much more efficient multiplication of integers in the ALU of the processor, and the complexity the multiplication operation goes from category $\mathrm{N}$ to $\log \mathrm{N}$. 


\begin{tabular}{|c|c|c|c|c|c|c|c|c|c|c|}
\hline & (32)00000000 & $(24) 00000000$ & (16)00000000 & (8)00000000 & $(128) 00000000$ & $(120) 00000000$ & (112)00000000 & $(104) 00000000$ & (96)00000000 & $(88) 0000000$ \\
\hline \multicolumn{11}{|l|}{ Factor a } \\
\hline \multicolumn{11}{|l|}{ Factor b } \\
\hline +Step1 & & \multicolumn{2}{|c|}{ A3*B6 } & \multicolumn{2}{|c|}{ A3*B4 } & \multicolumn{2}{|c|}{ A1*B4 } & & \multicolumn{2}{|c|}{$\mathrm{A} 4 * \mathrm{~B} 6$} \\
\hline +Step2 & & \multicolumn{2}{|c|}{$A 2 * B 7$} & \multicolumn{2}{|c|}{ A2*B5 } & \multicolumn{2}{|c|}{ A0*B5 } & & \multicolumn{2}{|c|}{$A 3 * B 7$} \\
\hline +Step3 & & & \multicolumn{2}{|c|}{$\mathrm{A} 2 * \mathrm{~B} 6$} & \multicolumn{2}{|c|}{$\mathrm{A} 0 * \mathrm{~B} 6$} & & \multicolumn{2}{|c|}{$\mathrm{A} 5 * \mathrm{~B} 6$} & \\
\hline +Step4 & & & \multicolumn{2}{|c|}{ A1*B7 } & \multicolumn{2}{|c|}{ A1*B5 } & & \multicolumn{2}{|c|}{$A 4 * B 7$} & \\
\hline +Step5 & & & & \multicolumn{2}{|c|}{$\mathrm{A} 1 * \mathrm{~B} 7$} & & \multicolumn{2}{|c|}{$A 6 * B 6$} & \multicolumn{2}{|c|}{$A 6 * B 4$} \\
\hline +Step6 & & & & \multicolumn{2}{|c|}{ A1*B6 } & & \multicolumn{2}{|c|}{$A 5 * B 7$} & \multicolumn{2}{|c|}{$A 5 * B 5$} \\
\hline +Step7 & \multicolumn{10}{|c|}{ An assembly step } \\
\hline +Step8 & & & & & & \multicolumn{2}{|c|}{$\mathrm{A} 7 * \mathrm{~B} 6$} & \multicolumn{2}{|c|}{ A7*B4 } & \\
\hline +Step9 & & & & & & \multicolumn{2}{|c|}{$A 6 * B 7$} & \multicolumn{2}{|c|}{$A 6 * B 5$} & \\
\hline +Step10 & & & & & \multicolumn{2}{|c|}{$A 7 * B 7$} & & B5 & \multicolumn{2}{|c|}{$A 7 * B 3$} \\
\hline RESULT & & & & & & & & & & \\
\hline
\end{tabular}

Fig.10. Optimization of 64-bit multiplication by using vacancies

This method of optimizing the operation of multiplication was considered for ALU, which has only one adder, but it is not an extremely optimal option. The summation sequence does not matter and therefore the steps of summing the blocks collected from the auxiliary multiplication table can be parallelized by using several adders. Thus, for an ALU with two adders, the operation of sequentially adding 10 numbers can be performed in 5 internal processors ticks (Fig.11). For ALU with 3 totalizers in 4 (Fig.12). Further increase in the number of totalizers more than 3 does not give an effect (Fig.13).

\begin{tabular}{|c|c|c|c|c|c|c|}
\hline & Adder1 & Adder2 & Adder2 & Adder1 & Adder1 & Adder2 \\
\hline +Step1 & \multirow[t]{2}{*}{ Step1' } & & \multirow[t]{4}{*}{ Step3" } & & \multirow[t]{10}{*}{ Step4" } & \multirow[t]{10}{*}{ Step5 } \\
\hline +Step2 & & & & & & \\
\hline +Step3 & & \multirow[t]{2}{*}{ Step1" } & & & & \\
\hline +Step4 & & & & & & \\
\hline +Step5 & \multirow[t]{2}{*}{ Step2' } & & & & & \\
\hline +Step6 & & & & & & \\
\hline +Step7 & & \multirow[t]{2}{*}{ Step2" } & & \multirow[t]{4}{*}{ Step4' } & & \\
\hline +Step8 & & & & & & \\
\hline +Step9 & \multirow[t]{2}{*}{ Step3' } & & & & & \\
\hline +Step10 & & & & & & \\
\hline
\end{tabular}

Fig.11 Implementation through 2 adders. 


\begin{tabular}{|c|c|c|c|c|c|c|c|}
\hline & Adder1 & Adder2 & Adder3 & Adder3 & Adder1 & Adder2 & Adder2 \\
\hline +Step1 & \multirow[t]{2}{*}{ Step1' } & & & \multirow[t]{4}{*}{ Step2"' } & & \multirow[t]{6}{*}{ Step3" } & Step4 \\
\hline +Step 2 & & & & & & & \\
\hline +Step3 & & \multirow[t]{2}{*}{ Step1" } & & & & & \\
\hline +Step4 & & & & & & & \\
\hline +Step5 & & & \multirow[t]{2}{*}{ Step1"' } & & & & \\
\hline +Step6 & & & & & & & \\
\hline +Step7 & & \multirow[t]{2}{*}{ Step2" } & & & \multirow[t]{4}{*}{ Step3' } & & \\
\hline +Step8 & & & & & & & \\
\hline +Step9 & \multirow[t]{2}{*}{ Step2' } & & & & & & \\
\hline +Step10 & & & & & & & \\
\hline
\end{tabular}

Fig.12 Implementation through 3 adders.

\begin{tabular}{|c|c|c|c|c|c|c|c|c|c|}
\hline & Adder1 & Adder2 & Adder3 & Adder4 & Adder1 & Adder2 & Adder3 & Adder4 & Adder1 \\
\hline +Step1 & \multirow[t]{2}{*}{ Step1' } & & & & & \multirow[t]{4}{*}{ Step2" } & & \multirow[t]{8}{*}{ Step3 } & \multirow[t]{10}{*}{ Step4 } \\
\hline +Step2 & & & & & & & & & \\
\hline +Step3 & & Step1" & & & & & & & \\
\hline +Step4 & & & & & & & & & \\
\hline +Step5 & & & \multirow[t]{2}{*}{ Step1"' } & & & & \multirow[t]{4}{*}{ Step2"' } & & \\
\hline +Step6 & & & & & & & & & \\
\hline +Step7 & & & & \multirow[t]{2}{*}{ Step1"'” } & & & & & \\
\hline +Step8 & & & & & & & & & \\
\hline +Step9 & & & & & \multirow[t]{2}{*}{ Step2' } & & & & \\
\hline +Step10 & & & & & & & & & \\
\hline
\end{tabular}

Fig.13 Implementation through 4 adders.

This article has shown an improved version of the integer multiplication, which spends less CPU time compared to the classic. 